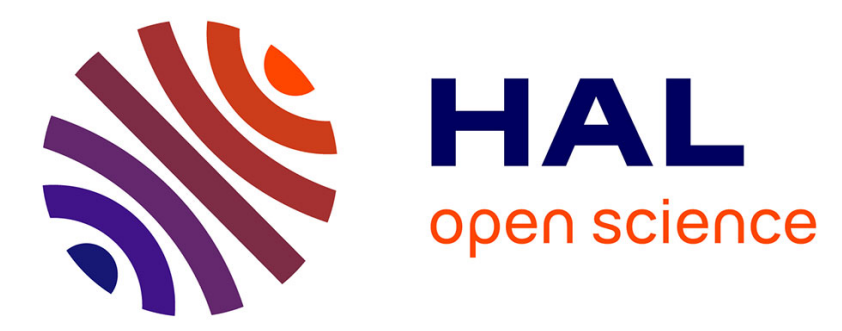

\title{
La CFTMEA R 2010, présentation des modifications de l'axe I
}

\author{
R. Misès, C. Bursztejn, M. Botbol, J. Garrabé, N. Garret Gloanec, B. Golse, \\ J.-P. Raynaud, G. Schmit, Y. Coincon, B. Durand, et al.
}

\section{- To cite this version:}

R. Misès, C. Bursztejn, M. Botbol, J. Garrabé, N. Garret Gloanec, et al.. La CFTMEA R 2010, présentation des modifications de l'axe I. Annales Médico-Psychologiques, Revue Psychiatrique, 2011, 169 (4), pp.248. 10.1016/j.amp.2011.03.006 . hal-00748145

\section{HAL Id: hal-00748145 \\ https://hal.science/hal-00748145}

Submitted on 5 Nov 2012

HAL is a multi-disciplinary open access archive for the deposit and dissemination of scientific research documents, whether they are published or not. The documents may come from teaching and research institutions in France or abroad, or from public or private research centers.
L'archive ouverte pluridisciplinaire HAL, est destinée au dépôt et à la diffusion de documents scientifiques de niveau recherche, publiés ou non, émanant des établissements d'enseignement et de recherche français ou étrangers, des laboratoires publics ou privés. 


\section{Accepted Manuscript}

Title: La CFTMEA R 2010, présentation des modifications de l'axe I

Authors: R. Misès, C. Bursztejn, M. Botbol, J. Garrabé, N. Garret Gloanec, B. Golse, J.-P. Raynaud, G. Schmit, Y. Coincon, B. Durand, Ch. Portelli, J.-P. Thévenot

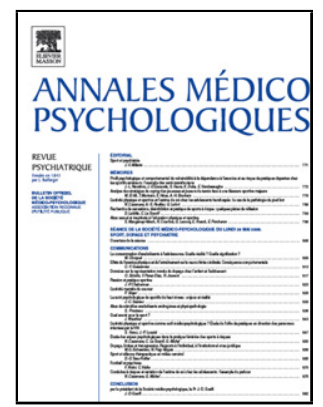

PII: S0003-4487(11)00071-0

DOI: doi:10.1016/j.amp.2011.03.006

Reference: AMEPSY 1313

To appear in: Annales Médico-Psychologiques

Please cite this article as: Misès R, Bursztejn C, Botbol M, Garrabé J, Gloanec NG, Golse B, Raynaud J-P, Schmit G, Coincon Y, Durand B, Portelli Ch, Thévenot JP, La CFTMEA R 2010, présentation des modifications de l'axe I, Annales mediopsychologiques (2010), doi:10.1016/j.amp.2011.03.006

This is a PDF file of an unedited manuscript that has been accepted for publication. As a service to our customers we are providing this early version of the manuscript. The manuscript will undergo copyediting, typesetting, and review of the resulting proof before it is published in its final form. Please note that during the production process errors may be discovered which could affect the content, and all legal disclaimers that apply to the journal pertain. 


\section{Communication}

\section{La CFTMEA R 2010, présentation des modifications de l'axe I}

The CFTMEA R 2010, new revision of the axis I

\section{R. Misès ${ }^{\text {a }}$, C. Bursztejn ${ }^{\text {b }}$ *, M. Botbol ${ }^{\text {c }}$, J. Garrabé ${ }^{\text {, }}$, N. Garret Gloanec ${ }^{\text {e }}$, B. Golse ${ }^{\text {f }}$,} J.-P. Raynaud ${ }^{\text {g }}$, G. Schmit ${ }^{\text {h }}$, Y. Coincon ${ }^{\mathrm{i}}$, B. Durand ${ }^{\mathrm{j}}$, Ch. Portelli ${ }^{\text {k }}$, J.-P. Thévenot ${ }^{1}$

a) Pr Roger Misès, 21 rue Barbet-de-Jouy, 75007, Paris, France

b) Pr Claude Bursztejn, CHRU Hôpital civil, 1 place de l’Hôpital, 67091 Strasbourg cedex, France

c) Dr Michel Botbol, Psychiatre des Hôpitaux, Clinique Dupré, 30 avenue F.-Roosevelt, 92331 Sceaux cedex, France

d) Dr Jean Garrabé, Psychiatre des Hôpitaux, 7, place Pinel, 75013 Paris, France

e) Dr Nicole Garret Gloanec, P.H., Centre nantais de la parentalité, 27, square La Pérouse, 44000 Nantes, France

f) Dr Bernard Golse, pédopsychiatre, hôpital Necker, 149 rue de Sèvres, 75743 Pais cedex 15, France

g) Dr Jean-Philippe Raynaud, CHU de Toulouse Hôpital La Grave, TSA 60033, 31059 Toulouse cedex 9, France

h) Dr Gérard Schmit, CHRU Hôpital R. Debré, avenue du Général Koenig, 51092 Reims cedex, France

i) Dr Yvonne Coinçon, pédopsychiatre, chef de secteur de psychiatrie infanto-juvénile

8 rue Servan 38000 Grenoble, France

j) Dr B. Durand, 121 avenue d'Italie, 75013 Paris, France

k) Dr Christian Portelli, Psychiatre des Hôpitaux, Médecin Directeur du CMPP de Boulogne, 12 rue Émile Landrin, 92100 Boulogne-Billancourt, France

l) Dr Jean-Pierre Thévenot, Psychiatre des Hôpitaux, CMP, 20 villa Compoint, 75017 Paris, France

* Auteur correspondant : Pr Claude Bursztejn, CHRU Hôpital civil, 1 place de l'Hôpital, 67091 Strasbourg cedex, France

Adresse email : claude.bursztejn@chru-strasbourg.fr 


\title{
Résumé
}

La CFTMEA, classification multiaxiale spécifique de la psychiatrie de l'enfant et de l'adolescent, se différencie du chapitre V de la CIM-10, ainsi que du DSM-IV, classifications syndromiques, par son organisation hiérarchisée.

L’axe I comporte cinq groupes de catégories principales mutuellement exclusives, correspondant à des structures psychopathologiques en référence aux conceptions psychodynamiques. Des catégories complémentaires permettant le recueil des éléments significatifs du tableau clinique sont à resituer dans l'une des organisations structurales.

Cette nouvelle révision (CFTMEA R 2010) a permis d'assurer une compatibilité pratiquement complète avec le chapitre V de la CIM-10, et de compléter les items proposés en catégorie complémentaire (ajout d'un chapitre), tout en conservant l'organisation d'ensemble de la classification.

Mots clés : Adolescent ; Classification ; Enfant ; Psychopathologie

\begin{abstract}
The French Classification of Child and Adolescent Psychiatry is a multiaxial diagnostic system specifically devoted to these ages. Unlike the $\mathrm{V}^{\text {th }}$ chapter of ICD-10 and the DSM-IV, which are syndromic classifications, it is organized on a hierarchical basis.

On axis I, five groups of principal categories are exclusive and correspond to psychopathological structures, in reference to psychodynamic concepts. Complementary categories are used to record significant clinical features, which have to be associated to one the psychopathological structure.

This new revision (CFTMEA R 2010) ensures an almost total compatibility with the $\mathrm{V}^{\text {th }}$ chapter of ICD-10; and brings more complementary items (one complete chapter has been added), while the general organization of the classification is maintained.
\end{abstract}

Keywords: Adolescent; Child; Classification; Psychopathology 
La publication du DSM-III, en 1980 [1], a marqué un tournant dans l’histoire des classifications que vient de rappeler Jean Garrabé : ses critères diagnostiques explicites, ses descriptions apparemment indépendantes de théorie étiopathogénique, son organisation multiaxiale, qui en faisait un guide clair et précis, l'efficacité enfin de sa diffusion, l’ont imposé au niveau international. La classification américaine a, depuis, exercé une influence indéniable sur les publications, ainsi que sur la $\mathrm{X}^{\mathrm{e}}$ édition de la Classification de l’OMS (CIM-10) $[8,9,11]$.

\section{Les classifications psychiatriques actuelles}

Comme on le sait, le DSM-IV [2], comme le chapitre V de la CIM-10, sont des classifications syndromiques. Pour ces classifications, la pertinence d'un syndrome est jugée sur la stabilité statistique du regroupement des signes caractéristiques - essentiellement comportementaux - et sur la fiabilité interjuge qu’assure sa description [9].

Si ces classifications se sont révélées efficaces dans le champ de la recherche en assurant la cohérence des diagnostics et en facilitant la communication scientifique, elles n’en ont pas moins suscité des critiques.

On doit d'abord noter qu'elles tendent à mettre sur le même plan des manifestations de nature très hétérogène. Ainsi, des organisations pathologiques (comme l'autisme ou la schizophrénie), des retards ou écarts par rapport à une norme développementale, des situations relationnelles (la rivalité fraternelle, item de la CIM-10), ou sociale (troubles des conduites), sont tous qualifiés de «trouble » (disorder) et critérisés de manière analogue.

D’autre part, il y a la prétention d’athéorisme, affirmée lors de la sortie du DSM-III, aujourd’hui largement contestée : il est assez évident que toutes ces catégories diagnostiques sont abordées selon un modèle biomédical, sans que cette référence soit explicitement justifiée. De fait, aucun principe de classification ne saurait être athéorique : la sélection de ce qui est considéré comme signe et le regroupement de manifestations en un syndrome implique des choix qui ne peuvent s’affranchir de préconceptions [9].

Enfin, la démarche clinique suggérée par ces classifications pose un problème épistémologique majeur : celui de la réduction de la clinique psychiatrique à des expressions manifestes, directement observables dans le comportement du patient. Cette position tend à accréditer l'idée discutable que les syndromes ainsi définis constituent des objets de 
connaissance cohérents, « détachables » du fonctionnement psychique et de l’histoire du sujet qui en souffre. Elle ne peut en outre s’accorder avec la démarche des cliniciens d'orientation psychodynamique, soucieux de prendre en compte la globalité du sujet dans la complexité de son fonctionnement psychique et de ses échanges avec son environnement.

Ces deux classifications générales soulèvent, en outre, d'autres questions dans le champ de la psychiatrie infanto-juvénile: elles ne proposent en effet qu'un nombre limité d’items spécifiques pour l'enfant. Dans ces items, on retrouve les catégories décrites par les initiateurs de la psychiatrie de l'enfant, au début du $\mathrm{xx}^{\mathrm{e}}$ siècle, à partir de données purement symptomatiques: à savoir des troubles des conduites, des troubles anxieux, des troubles oppositionnels, etc.

En dehors de ces quelques catégories diagnostiques, le DSM comme la CIM recommandent d'utiliser les mêmes catégories et les mêmes critères chez l'enfant et chez l'adulte: ainsi se sont développées des recherches sur la dépression, les troubles obsessionnels, les troubles anxieux chez l'enfant, abordés à partir des critères de la pathologie adulte. Cette approche conduit à assimiler abusivement des manifestations transitoires avec des syndromes fixés ; elle tend à privilégier les manifestations cliniques qui, au cours de l'enfance, annoncent une pathologie au long cours, au détriment d'autres aspects cliniques non moins importants, notamment ceux qui sont liés au développement [3] ; elle néglige enfin la part du contexte interrelationnel dans l'émergence des symptômes présentés par un enfant.

Au cours des dernières décennies, les pédopsychiatres français se sont au contraire attachés à étudier les aspects les plus spécifiques de la psychopathologie dans l'enfance, et ont dégagé des repères susceptibles d'éclairer la clinique qu'ils observent, dans le cadre de suivis thérapeutiques de longue durée - qu’il s’agisse de psychothérapies individuelles ou de prises en charge institutionnelles.

Les composantes multifactorielles présentes dans l'avènement et l'évolution des perturbations survenant dans l'enfance et l'adolescence ont ainsi été reconnues.

Ces orientations ont été à l'origine de la mise au point d'une Classification des Troubles mentaux de l'Enfant et de l'Adolescent (CFTMEA) susceptible de rencontrer l'adhésion des praticiens, quelles que soient leurs options théoriques, dès lors qu'ils pouvaient s’accorder sur des points fondamentaux de la clinique [4-7]. 


\section{La CFTMEA}

La CFTMEA, dont la première édition a été publiée sous la direction de R. Misès en 1988 [4], a systématisé les travaux nosographiques et psychopathologiques élaborés au cours du développement de la pédopsychiatrie dans notre pays. Contrairement aux classifications qui tendent à réduire la démarche diagnostique à une addition de critères comportementaux, la CFTMEA affirme l'importance d'une démarche clinique et psychopathologique s'appuyant sur les approches psychodynamiques.

Elle privilégie la notion de structure psychopathologique à entendre comme un ensemble de positions libidinales, de types d'angoisses et de mécanismes défensifs interdépendants relativement stables mais susceptibles de remaniements évolutifs en fonction de la maturation psychique mais aussi des interventions thérapeutiques.

La CFTMEA s’ordonne autour de deux axes, l'axe I des «catégories cliniques de base », et l'axe II des « facteurs antérieurs, éventuellement étiologiques », ce dernier étant luimême subdivisé en deux rubriques, l'une pour les atteintes organiques, l'autre pour les conditions d'environnement. Il en résulte que le clinicien est incité à séparer assez clairement le temps, prioritaire, de la recherche d'une catégorie clinique sur l'axe $\mathrm{I}$, de l'autre temps, où il fait l'inventaire des facteurs appelés à figurer sur l'axe II. Cette procédure contribue à éviter les raccourcis étiopathogéniques à visée explicative, et elle conduit à effectuer un inventaire assez large d'où résulte la mise en valeur de facteurs multiples, susceptibles d'entrer en interrelation mutuelle. Cette démarche vise à échapper à la pesée des idéologies contraignantes concernant le déterminisme univoque des troubles.

Les quatre premiers chapitres de la CFTMEA (Tableau 2) se réfèrent à la notion de structure psychopathologique évoquée précédemment. Il est demandé au clinicien de préciser l'organisation psychopathologique dans laquelle s’inscrivent les symptômes présentés par l'enfant, par l'une des catégories proposées dans ces chapitres :

- autisme et psychoses ;

- troubles névrotiques ;

- pathologies de la personnalité ;

- troubles réactionnels.

À ces quatre catégories principales, il faut ajouter le groupe des variations de la 
normale, apport original de cette classification, qui permet de rendre compte des symptômes transitoires pouvant survenir au cours du développement, susceptibles d'être à l'origine de consultations, sans qu'il soit justifié de leur affecter une signification pathologique. Cependant, la CFTMEA ne néglige pas le recueil précis de la symptomatologie, important pour les orientations thérapeutiques : des chapitres complémentaires (Tableau 3) proposent des items permettant de recueillir les éléments significatifs du tableau clinique. Les troubles fonctionnels ou symptômes qui sont regroupés dans cette partie sont des éléments diagnostiques secondaires à resituer dans l'une des organisations structurales.

Ainsi conçue, la CFTMEA constitue une classification statistique, biaxiale, dotée d'un glossaire qui précise les critères d’inclusion et d'exclusion. Les essais effectués [10] en ont montré la facilité d'utilisation, la maniabilité et son adéquation aux orientations dominantes de la pédopsychiatrie en France.

C’est cette classification qui a été intégrée dans la « fiche par patient » mise en place par la Direction générale de la Santé pour le recueil des activités des secteurs de psychiatrie infanto-juvénile [10]. Elle a pu être utilisée dans la première version du PMSI psychiatrie (selon un texte de juin 2002 qui figure encore sur le site de l'ATIH : http://www.atih.sante.fr/?id=0002F00059FF).

Cependant, sa place de classification de référence est menacée par la mise en place de la deuxième version du PMSI en psychiatrie (RIMPSY), pour lequel l'administration a décidé d'imposer le seul codage selon la CIM-10.

\section{La révision de la CFTMEA}

La CFTMEA, comme toutes les classifications, a connu plusieurs révisions : l'une des plus importantes - CFTMEA R2000 - a été présentée à la Société Médico-Psychologique [5]. Les modifications apportées visaient à mieux prendre en compte certains aspects de la clinique jusque-là moins bien étudiés. Dès sa première version, il est apparu nécessaire à ses auteurs d'établir le plus de correspondances possibles entre les diagnostics décrits par la CFTMEA et ceux de la Classification de l'OMS.

Malgré les différences conceptuelles entre ces deux classifications, des tableaux d’équivalence ont pu être établis. Ils ont été progressivement améliorés de version en version.

La décision évoquée plus haut d’imposer pour le PMSI le codage des diagnostics en 
référence à la CIM-10, en psychiatrie de l'enfant comme en psychiatrie de l'adulte, a rendu nécessaire une révision approfondie des équivalences entre la classification de l’OMS et la CFTMEA : il s'agissait de permettre aux praticiens, qui en majorité le souhaitaient, de continuer d'utiliser un cadre conceptuel le mieux adapté à leurs pratiques et à leurs conceptions théoriques.

Dans un premier temps, un groupe de travail (C. Bursztejn, Y. Coinçon, C. Portelli, J.P. Bodin) a repris et complété les tableaux d’équivalences.

Mais il est apparu que pour certains diagnostics proposés par la CFTMEA, il n'était pas possible de trouver une correspondance simple, soit parce que la CIM-10 était plus détaillée que la classification francophone dans le domaine considéré de la nosographie, soit parce que la logique du codage était différente (c’était le cas par exemple du retard mental).

Cela obligeait à recourir aux deux classifications, opération complexe et fastidieuse dans le contexte de la pratique quotidienne.

C’est ce qui a conduit à la proposition d'une nouvelle révision, qui tout en maintenant la conception structurale de la CFTMEA, permettait une mise en correspondance terme à terme la plus complète possible avec les codes de la CIM-10.

\section{CFTMEA R-2010 : principales modifications}

Les modifications les plus importantes des catégories de l'axe I sont les suivantes :

- troubles schizophréniques de l’adolescence ne comportant que deux sous-catégories : « aspects prodromiques » et "schizophrénie avérée »; dans la nouvelle version ont été reprises les différentes formes syndromiques décrites de la CIM-10 ;

- retard mental : le codage du deuxième caractère, qui dans la CIM-10 indique le degré de retentissement fonctionnel, a été repris ;

- troubles liés à l'usage de drogues ou d'alcool : le codage de la CIM-10 a été intégralement repris, à la fois pour les subdivisions selon les substances et pour le type de trouble mental lié à l’abus de substance.

Enfin, il est apparu nécessaire de compléter les items proposés en catégorie complémentaire, dans un double objectif : assurer une meilleure compatibilité avec la CIM, mais aussi permettre de relever certains symptômes fréquents - anxieux, conversifs, phobiques ou compulsifs, susceptibles de survenir dans différents contextes 
psychopathologiques (pathologies névrotiques, ou limites notamment). Cela a nécessité d'ajouter un chapitre (numéro 9).

Du fait de cet ajout, le codage des variations de la normale - un groupe particulièrement important en pratique clinique ambulatoire de pédopsychiatrie - se trouve décalé ; il a été affecté du code zéro, et logiquement regroupé avec les catégories principales.

Enfin, il est apparu nécessaire de réviser l'axe II afin d'en permettre le transcodage vers la CIM-10.

\section{Conclusion}

Au total, on voit qu'il n’a été apporté que des modifications mineures aux catégories principales auxquelles doit se référer le diagnostic structural : les changements les plus importants portent sur les chapitres regroupant le codage des éléments symptomatiques et descriptifs d'une observation.

Ces modifications respectent ainsi la conception qui a été celle de la CFTMEA depuis son origine, mais elles permettent désormais un transcodage aisé vers la CIM-10, transcodage qu'il est possible d'automatiser dans des logiciels de traitement des informations RIMPSY afin de répondre aux exigences administratives et statistiques.

Cette amélioration du transcodage va aussi dans le sens d'une facilitation de l'accès des travaux cliniques des équipes françaises aux publications internationales, pour lesquelles la référence à la classification internationale est une exigence incontournable.

\section{Conflit d'intérêt : à compléter par l'auteur}

\section{Références}

[1] American Psychiatric Association. Diagnostic and Statistical Manual of Mental Disorders (DSM-III). Washington: American Psychiatric Association; 1980.

[2] American Psychiatric Association. Diagnostic and Statistical Manual of Mental Disorders - Revised (DSM-IV). Washington: American Psychiatric Association; 1994.

[3] Bursztejn C, Mazet Ph. Nouvelles classifications en psychiatrie de l'enfant. Éditions Techniques Encycl. Méd. Chir., Psychiatrie 1991, 37200 B $^{10}$.

[4] Misès R, Fortineau J, Jeammet Ph, Lang JL, Mazet Ph, Plantade A, Quemada N. 
Classification française des troubles mentaux de l'enfant et de l'adolescent. Psychiatrie de l’enfant 1988;31:67-134.

[5] Misès R, Quemada N, Botbol $M$, Bursztejn C, Durand B, Garrabé J, et al. CFTMEA 2000 : nouvelle version de la Classification française des troubles mentaux de l'enfant et de l'adolescent. Ann Med Psychol 2002;160:213-5.

[6] Misés R, Garret-Gloanec N, Coinçon Y. Classification de l'autisme et des psychoses précoces, plaidoyer pour des convergences. L’Information Psychiatrique 2010;85:223-6.

[7] Mises R, Quemada N, Botbol M, Bursztejn C, Garrabe J, Golse B, et al. French Classification for Child and Adolescent Mental Disorders. Psychopathology 2002;35:176-80.

[8] OMS. CIM-10/ICD-10. Classification Internationale des Troubles Mentaux et des Troubles du Comportement. Paris: Masson; 1993.

[9] Pedinielli JL, Bertagne P, Gimenez G. Classifications syndromiques et classifications psychopathologiques. L’information Psychiatrique 1999;75:289-95.

[10] Quemada N. L'utilisation de la classification française des troubles mentaux de l'enfant et de l'adolescent dans une enquête nationale. Neuropsychiatrie de l'enfance et de l'adolescence 1990;38:540-5.

[11] Sartorius N. Classification des troubles mentaux selon la CIM-10. L’Encéphale 1995;21:9-13.

\section{Discussion}

Dr C. Houssou - Comment la CFTMEA aborde-t-elle l'approche diachronique dans l'utilisation en pratique de la CFTMEA?

La CFTMEA propose-t-elle des directives ou des éléments différenciant le diagnostic principal du ou des diagnostics secondaires chez un patient?

$\operatorname{Pr}$ C. Barrois - Il a été question ici de « structures ». Je sais que c’est un vieux serpent de mer mais, le concept de structure, en psychiatrie, présente-t-il une solidité et une pertinence suffisantes ? Est-il justiciable d’une définition opératoire acceptable pour les cliniciens ?

Note de la secrétaire de rédaction : le tableau 1 est joint à cet article. A fortiori les deux 
tableaux ci-dessous s’appelleront tableau 2 et tableau 3. Mais je n’ai pas su modifier l'intitulé dans le cadre de ces deux tableaux. 


\title{
Tableau 1
}

\author{
CFTMEA - Révision 2010
}

Table de correspondance avec la Cim-10 (avec intitulés CIM 10)

\begin{tabular}{|c|c|c|c|}
\hline & INTITULES CFTMEA & $\begin{array}{l}\text { Codes } \\
\text { Cim-10 }\end{array}$ & INTITULES CIM \\
\hline 0 & VARIATIONS DE LA NORMALE & & \\
\hline 0.0 & ANGOISSES, RITUELS, PEURS & F93.1 & Troubles anxieux phobique de l'enfance \\
\hline 0.1 & MOMENTS DÉPRESSIFS & F38.0 & $\begin{array}{l}\text { Autres troubles de l'humeur [affectifs] } \\
\text { isolés }\end{array}$ \\
\hline 0.2 & CONDUITES D'OPPOSITION & F91.8 & Autres troubles des conduites \\
\hline 0.3 & CONDUITES D'ISOLEMENT & F93.1 & Troubles anxieux phobique de l'enfance \\
\hline 0.4 & $\begin{array}{c}\text { DIFFICULTÉS SCOLAIRES NON CLASSABLES } \\
\text { AILLEURS }\end{array}$ & Z55.4 & $\begin{array}{l}\text { Difficultés liées à une mauvaise } \\
\text { adaptation éducative et difficultés avec } \\
\text { les enseignants et les autres élèves }\end{array}$ \\
\hline 0.5 & RETARDS OU RÉGRESSIONS TRANSITOIRES & R62.8 & $\begin{array}{c}\text { Autres retards du développement } \\
\text { physiologique }\end{array}$ \\
\hline 0.6 & ASPECTS ORIGINAUX DE LA PERSONNALITÉ & R46.8 & $\begin{array}{l}\text { Autres symptômes et signes relatifs à } \\
\text { l'apparence et au comportement }\end{array}$ \\
\hline 1 & \multicolumn{3}{|c|}{ AUTISME ET TROUBLES PSYCHOTIQUES } \\
\hline 1.0 & \multicolumn{3}{|c|}{ TROUBLES ENVAHISSANTS DU DÉVELOPPPEMENT (TED) - PSYCHOSES PRÉCOCES } \\
\hline 1.00 & Autisme infantile précoce - type Kanner & F84.0 & Autisme infantile \\
\hline 1.01 & Autres formes de l'autisme & F84.1 & Autisme atypique \\
\hline 1.02 & $\begin{array}{l}\text { Psychose précoce déficitaire. Retard mental avec } \\
\text { troubles autistiques ou psychotiques } \\
\text { (et préciser le retard mental cf chap 5) }\end{array}$ & $\begin{array}{c}\text { F84.1 } \\
+ \\
\text { F70- } \\
\text { F79 }\end{array}$ & $\begin{array}{c}\text { Autisme atypique } \\
+ \\
\text { Retard mental }\end{array}$ \\
\hline 1.03 & Syndrome d'Asperger & F84.5 & Syndrome d'Asperger \\
\hline
\end{tabular}




\begin{tabular}{|c|c|c|c|}
\hline 1.04 & $\begin{array}{c}\text { Dysharmonie Multiple et Complexe du } \\
\text { Développement (MCDD) - (Dysharmonie } \\
\text { psychotique) }\end{array}$ & F84.8 & Autres TED \\
\hline 1.05 & Troubles désintégratifs de l'enfance & F84.3 & Autre trouble désintégratif de l'enfance \\
\hline 1.08 & Autres formes précoces & F84.8 & Autres TED \\
\hline 1.09 & Formes précoces NS & F84.9 & TED, sans précision \\
\hline 1.1 & \multicolumn{3}{|c|}{ SCHIZOPHRÉNIES } \\
\hline 1.10 & Schizophrénie de l'enfant & F20.9 & Schizophrénie, sans précision \\
\hline 1.11 & Tr. schizophrénique. à l'adolescence & F20.9 & Schizophrénie, sans précision \\
\hline 1.110 & - Aspects prodromiques & F21 & Trouble schizotypique \\
\hline 1.111 & - Schizophrénie paranoïde & F20.0 & Schizophrénie paranoïde \\
\hline 1.112 & - Schizophrénie hébéphrénique & F20.1 & Schizophrénie hébéphrénique \\
\hline 1.113 & - Schizophrénie catatonique & F20.2 & Schizophrénie catatonique \\
\hline 1.114 & - Schizophrénie indifférenciée & F20.3 & Schizophrénie indifférenciée \\
\hline 1.115 & - Dépression post-schizophrénique & F20.4 & Dépression post-schizophrénique \\
\hline 1.116 & - Schizophrénie résiduelle & F20.5 & Schizophénie résiduelle \\
\hline 1.117 & - Schizophrénie simple & F20.6 & Schizophrénie simple \\
\hline 1.118 & - Autres formes de schizophrénie & F20.8 & Autres formes de schizophrénie \\
\hline 1.119 & - Schizophrénie sans précision & F20.9 & Schizophrénie, sans précision \\
\hline 1.2 & TR. DÉLIRANTS & F22.8 & Autres troubles délirants persistants \\
\hline 1.3 & TR. PSYCHOTIQUES AIGUS & & \\
\hline 1.30 & $\begin{array}{l}\text { Trouble psychotique aigu polymorphe sans } \\
\text { symptômes schizophréniques }\end{array}$ & F23.0 & $\begin{array}{l}\text { Trouble psychotique aigu polymorphe, sans symptômes } \\
\text { schizophréniques }\end{array}$ \\
\hline 1.31 & $\begin{array}{l}\text { Trouble psychotique aigu polymorphe avec } \\
\text { symptômes schizophréniques }\end{array}$ & F23.1 & $\begin{array}{l}\text { Trouble psychotique aigu polymorphe avec symptômes } \\
\text { schizophréniques }\end{array}$ \\
\hline 1.38 & Autres troubles psychotiques aigus & F23.8 & Autres troubles psychotiques aigus et transitoires \\
\hline
\end{tabular}




\begin{tabular}{|c|c|c|c|}
\hline 1.4 & TR. THYMIQUES & & \\
\hline 1.40 & Psychoses dysthymiques de l'enfant & $\begin{array}{r}F 84.8 \\
+F 34.0\end{array}$ & $\begin{aligned} & \text { Autres TED } \\
+ & \text { Cyclothymie }\end{aligned}$ \\
\hline 1.41 & Troubles thymiques de l'adolescent & F25.9 & Trouble schizo-affectif, sans précision \\
\hline 1.410 & - Épisode maniaque & F30.9 & Épisode maniaque, sans précision \\
\hline 1.4100 & o EM actuel s'inscrivant dans un trouble bipolaire & F31.0 & Trouble affectif bipolaire, épisode actuel hypomaniaque \\
\hline 1.4101 & o sans symptômes psychotiques & F30.1 & Manie sans symptômes psychotiques \\
\hline 1.4102 & o avec symptômes psychotiques & F30.2 & Manie avec symptômes psychotiques \\
\hline 1.4103 & o Etat mixte & F38.8 & Autres troubles de l'humeur [affectifs] précisés \\
\hline 1.4104 & o Hypomanie & F30.0 & Hypomanie \\
\hline 1.411 & - Épisode dépressif (ED) & F32.9 & Épisode dépressif, sans précision \\
\hline 1.4110 & oED actuel dans un tr bipolaire & F31.4 & $\begin{array}{c}\text { Trouble affectif bipolaire, épisode actuel de dépression } \\
\text { sévère sans symptômes psychotiques }\end{array}$ \\
\hline 1.4111 & oED sévère non mélancolique & F32.8 & Autres épisodes dépressifs \\
\hline 1.4112 & $\begin{array}{c}\text { oED sévère non mélancolique avec symptômes } \\
\text { psychotiques }\end{array}$ & F32.3 & $\begin{array}{l}\text { Épisode dépressif sévère avec symptômes } \\
\text { psychotiques }\end{array}$ \\
\hline 1.4113 & oED sévère mélancolique & F32.2 & $\begin{array}{c}\text { Épisode dépressif sévère sans symptômes } \\
\text { psychotiques }\end{array}$ \\
\hline 1.4114 & oED sévère avec mélancolie délirante & F32.3 & $\begin{array}{l}\text { Épisode dépressif sévère avec symptômes } \\
\text { psychotiques }\end{array}$ \\
\hline 1.5 & $\begin{array}{l}\text { ÉTATS DÉPRESSIFS APRÈS ÉPISODE } \\
\text { PSYCHOTIQUE }\end{array}$ & F20.4 & Dépression post-schizophrénique \\
\hline 1.8 & AUTRES TR. PSYCHOTIQUES & F28 & $\begin{array}{c}\text { Autres troubles psychotiques non } \\
\text { organiques }\end{array}$ \\
\hline 1.9 & TR. PSYCHOTIQUES NON SPÉCIFIÉS & F29 & Psychose non organique, sans précision \\
\hline
\end{tabular}

\begin{tabular}{|c|c|c|c|}
\hline 2 & TR. NEVROTIQUES & \\
\hline 2.0 & TR. NÉVROTIQUES À DOMINANTE ANXIEUSE & F41.9 & Trouble anxieux, sans précision \\
\hline 2.1 & TR. NÉVROTIQUES Ȧ DOMINANTE HYSTÉRIQUE & $\mathbf{F 4 4 . 9}$ & $\begin{array}{c}\text { Trouble dissociatif [de conversion], sans } \\
\text { précision }\end{array}$ \\
\hline 2.2 & $\begin{array}{c}\text { T. NÉVROTIQUES À DOMINANTE PHOBIQUE } \\
2.3\end{array}$ & $\mathbf{F 4 0 . 9}$ & \begin{tabular}{c} 
Trouble anxieux phobique, sans précision \\
\hline
\end{tabular} \\
\hline
\end{tabular}




\begin{tabular}{|c|c|c|c|}
\hline 2.4 & $\begin{array}{c}\text { TR. NÉVROTIQUES AVEC PRÉDOMINANCE DES } \\
\text { INHIBITIONS }\end{array}$ & $\begin{array}{c}\mathbf{F 4 8 . 8} \\
\mathbf{+} \mathbf{R 4 6 . 4}\end{array}$ & $\begin{array}{c}\text { Autres troubles névrotiques précisés } \\
+ \text { Lenteur et manque de réactivité }\end{array}$ \\
\hline 2.5 & DÉPRESSION NÉVROTIQUE & $\mathbf{F 4 8 . 8}$ & $\begin{array}{c}\text { Autres troubles névrotiques précisés } \\
+ \text { Épisode dépressif, sans précision }\end{array}$ \\
\hline 2.6 & $\begin{array}{c}\text { CARACTĖRES NÉVROTIQUES, PATHOLOGIES } \\
\text { NÉVROTIQUES DE LA PERSONNALITÉ }\end{array}$ & $\mathbf{F 4 8 . 8}$ & $\begin{array}{c}\text { Autres troubles névrotiques précisés } \\
+ \text { Trouble de la personnalité, sans } \\
\text { précision }\end{array}$ \\
\hline 2.7 & $\begin{array}{c}\text { TR. NÉVROTIQUES AVEC PERTURBATIONS } \\
\text { PRÉDOMINANTES DES FONCTIONS } \\
\text { INSTRUMENTALES (pour préciser les troubles } \\
\text { instrumentaux cf chap 6) }\end{array}$ & $\mathbf{F 4 8 . 8}$ & Autres troubles névrotiques précisés \\
\hline 2.8 & $\begin{array}{c}\text { TR. NÉVROTIQUES À EXPRESSION PLURIMODALE } \\
\text { + T48.8 }\end{array}$ & $\mathbf{F 4 8 . 9}$ & Autres troubles névrotiques précisés \\
\hline
\end{tabular}




\begin{tabular}{|c|c|c|c|}
\hline \multicolumn{3}{|c|}{ PATHOLOGIES LIMITES } & \\
\hline 3.0 & $\begin{array}{c}\text { DYSHARMONIES ÉVOLUTIVES } \\
\text { (pour préciser les éventuels troubles instrumentaux } \\
\text { cf chap 6) }\end{array}$ & F60.3 & Personnalité émotionnellement labile \\
\hline 3.1 & $\begin{array}{c}\text { PATHOLOGIE LIMITE À DOMINANTE DES TR. DE LA } \\
\text { PERSONNALITÉ }\end{array}$ & F92.8 & $\begin{array}{l}\text { Autres troubles mixtes des conduites et } \\
\text { troubles émotionnels }\end{array}$ \\
\hline 3.2 & $\begin{array}{l}\text { PATHOLOGIE LIMITE À DOMINANTE } \\
\text { SCHIZOTYPIQUE }\end{array}$ & F21 & Trouble schizotypique \\
\hline 3.3 & $\begin{array}{l}\text { PATHOLOGIE LIMITE À DOMINANTE } \\
\text { COMPORTEMENTALE }\end{array}$ & F91.9 & Trouble des conduites, sans précision \\
\hline 3.4 & DÉPRESSIONS LIÉES À UNE PATHOLOGIE LIMITE & F92.0 & Troubles des conduites avec dépression \\
\hline 3.8 & AUTRES PATHOLOGIES LIMITES & F98.8 & $\begin{array}{l}\text { Autres troubles précisés du } \\
\text { comportement et troubles émotionnels } \\
\text { apparaissant habituellement durant } \\
\text { l'enfance et l'adolescence }\end{array}$ \\
\hline 3.9 & PATHOLOGIE LIMITE NS & F98.9 & $\begin{array}{c}\text { Trouble du comportement et trouble } \\
\text { émotionnel apparaissant habituellement } \\
\text { durant l'enfance et l'adolescence, sans } \\
\text { précision }\end{array}$ \\
\hline
\end{tabular}

\begin{tabular}{|c|c|c|c|}
\hline 4 & TROUBLES REACTIONNELS & \\
\hline 4.0 & DÉPRESSION RÉACTIONNELLE & $\begin{array}{c}\text { F43.2 } \\
+\mathbf{F 3 2 . 9}\end{array}$ & $\begin{array}{c}\text { Troubles de l'adaptation } \\
+ \text { Épisode dépressif, sans précision }\end{array}$ \\
\hline 4.1 & MANIFESTATIONS RÉACTIONNELLES & $\mathbf{F 4 3 . 2}$ & Troubles de l'adaptation \\
\hline 4.2 & SYNDROME DE STRESS POST-TRAUMATIQUE & $\mathbf{F 4 3 . 1}$ & État de stress posttraumatique \\
\hline
\end{tabular}

\begin{tabular}{|c|c|c|c|}
\hline \multicolumn{3}{|c|}{ DEFICIENCES MENTALES } & \\
\hline 5.0 & Q1 $50-69$ & F70.- & Retard mental léger* $^{*}$ \\
\hline 5.1 & Q $35-49$ & F71.- & Retard mental moyen* \\
\hline 5.2 & QI $20-34$ & F72.- & Retard mental grave* \\
\hline 5.3 & QI $<20$ & F73.- & Retard mental profond* \\
\hline
\end{tabular}




\begin{tabular}{|c|c|c|c|}
\hline 5.4 & QI Non Spécifié & F79.- & Retard mental, sans précision* \\
\hline & & $\begin{array}{l}.0 \\
.1 \\
.8 \\
.9\end{array}$ & $\begin{array}{l}\text { *Préciser : } \\
\text {-1 déficience du comportement absent ou minime } \\
\text {-2 déficience du comportement significatif, nécessitant } \\
\text { surveillance ou traitement } \\
\text {-3 autres déficiences du comportement } \\
\text { sans mention d'une déficience du comportement }\end{array}$ \\
\hline $5 . \times 6$ & $\begin{array}{c}\text { Déficiences dysharmoniques } \\
\text { (ajouter le code au type de pathologie limite } \\
\text { associée cf chap 3, et coder le retard mental) }\end{array}$ & & \\
\hline $5 . x 7$ & $\begin{array}{c}\text { Déficience avec polyhandicap sensoriel et/ou } \\
\text { moteur } \\
\text { (ajouter le code du/des handicaps associés) }\end{array}$ & & \\
\hline $5 . \times 8$ & Démences & F03 & Démence, sans précision \\
\hline $5 . \times 9$ & Non spécifiées & F79.9 & $\begin{array}{l}\text { Retard mental, sans précision : sans mention d'une } \\
\text { déficience du comportement }\end{array}$ \\
\hline
\end{tabular}

\begin{tabular}{|c|c|c|c|}
\hline 6 & \multicolumn{3}{|c|}{ TROUBLES DU DÉVELOPPEMENT ET DES FONCTIONS INSTRUMENTALES } \\
\hline 6.0 & TR. DE LA PAROLE ET DU LANGAGE & & \\
\hline 6.00 & Troubles isolés de l'articulation & F80.0 & Trouble spécifique de l'acquisition de l'articulation \\
\hline 6.01 & Troubles du développement du langage & F80.9 & $\begin{array}{c}\text { Trouble du développement de la parole et du langage, } \\
\text { sans précision }\end{array}$ \\
\hline 6.010 & - Retard de parole & F80.1 & Trouble de l'acquisition du langage, de type expressif \\
\hline 6.011 & - Retard simple de langage & F80.1 & Trouble de l'acquisition du langage, de type expressif \\
\hline 6.012 & - Dysphasie & F80.2 & Trouble de l'acquisition du langage, de type réceptif \\
\hline 6.018 & - Autres tr. du développement.du langage & F80.8 & $\begin{array}{l}\text { Autres troubles du développement de la parole et du } \\
\qquad \text { langage }\end{array}$ \\
\hline 6.02 & Aphasie acquise & & \\
\hline 6.020 & - Aphasie acquise avec épilepsie & F80.3 & Aphasie acquise avec épilepsie [Landau-Kleffner] \\
\hline 6.028 & - Autres aphasies acquises & R47.02 & Aphasie, autres et sans précision \\
\hline 6.03 & Mutisme & & \\
\hline 6.030 & - Mutisme total & F94.8 & Mutisme complet \\
\hline 6.031 & - Mutisme électif & F94.0 & Mutisme électif \\
\hline 6.04 & Bégaiement & F98.5 & Bégaiement \\
\hline 6.08 & Autres tr. de la parole et du langage & R47.8 & Troubles du langage, autres et non précisés \\
\hline 6.09 & Tr. de la parole et du langage NS & F80.9 & $\begin{array}{l}\text { Trouble du développement de la parole et du langage, } \\
\text { canc nróricion }\end{array}$ \\
\hline
\end{tabular}




\begin{tabular}{|c|c|c|c|}
\hline & & & sans précision \\
\hline 6.1 & TR. COGNITIFS ET DES ACQUISITIONS SCOLAIRES & & \\
\hline 6.10 & Tr. Lexicographiques & & \\
\hline 6.100 & - Dyslexie isolée & F81.0 & Trouble spécifique de la lecture \\
\hline 6.101 & - Tr. de l'orthographe isolé & F81.1 & Trouble spécifique de l'acquisition de l'orthographe \\
\hline 6.108 & - Autres tr. lexicographiques & F81.8 & $\begin{array}{l}\text { Autres troubles du développement, des acquisitions } \\
\text { scolaires }\end{array}$ \\
\hline 6.11 & Tr. spécifiques de l'arithmétique (dyscalculie) & F81.2 & Trouble spécifique de l'acquisition de l'arithmétique \\
\hline 6.12 & Tr. du raisonnement (dysharmonies cognitives) & F88 & Autres troubles du développement psychologique \\
\hline 6.13 & Tr. de l'attention sans hyperkinésie & F81.8 & $\begin{array}{l}\text { Autres troubles du développement, des acquisitions } \\
\qquad \text { scolaires }\end{array}$ \\
\hline 6.18 & Autres tr. cognitifs \& des acquisitions scolaires & F81.8 & $\begin{array}{l}\text { Autres troubles du développement, des acquisitions } \\
\text { scolaires }\end{array}$ \\
\hline 6.19 & Tr. cognitifs \& des acquisitions scolaires NS & F81.9 & $\begin{array}{l}\text { Trouble du développement, des acquisitions scolaires, } \\
\text { sans précision }\end{array}$ \\
\hline 6.2 & TR. PSYCHOMOTEURS & & \\
\hline 6.20 & $\begin{array}{l}\text { Retard psychomoteur (tr. spécifiques du } \\
\text { développement moteur) }\end{array}$ & F82 & Trouble spécifique du développement moteur \\
\hline 6.21 & Tics & F95.9 & Tic, sans précision \\
\hline 6.210 & - Tics isolés transitoires & F95.0 & Tic transitoire \\
\hline 6.211 & - Tic moteur ou vocal chronique & F95.1 & Tic moteur ou vocal chronique \\
\hline 6.212 & - Maladie de Gilles de la Tourette & F95.2 & $\begin{array}{l}\text { Forme associant tics vocaux et tics moteurs [syndrome } \\
\text { de Gilles de la Tourette] }\end{array}$ \\
\hline 6.28 & Autres tr. psychomoteurs & F83 & Troubles spécifiques mixtes du développement \\
\hline 6.29 & Tr. psychomoteurs NS & R62.8 & Autres retards du développement physiologique \\
\hline
\end{tabular}

\begin{tabular}{|c|c|c|c|}
\hline \multicolumn{4}{|c|}{ TROUBLES DES CONDUITES ET DU COMPORTEMENT } \\
\hline 7.0 & TR. HYPERKINÉTIQUES & & \\
\hline 7.00 & Hyperkinésie avec troubles de l'attention & $\mathrm{F} 90.0$ & Perturbation de l'activité et de l'attention \\
\hline 7.08 & Autres tr. hyperkinétiques & $\mathrm{F} 90.8$ & Autres troubles hyperkinétiques \\
\hline 7.09 & Tr. hyperkinétiques NS & $\mathrm{F} 90.9$ & Trouble hyperkinétique, sans précision \\
\hline 7.1 & TR. DES CONDUITES ALIMENTAIRES & & \\
\hline 7.10 & Anorexie mentale & 50.0 & \\
\hline
\end{tabular}




\begin{tabular}{|c|c|c|c|}
\hline 7.100 & - Anorexie mentale restrictive & F50.0 & Anorexie mentale \\
\hline 7.101 & - Anorexie mentale boulimique & $\begin{array}{r}F 50.0 \\
+F 50.2\end{array}$ & $\begin{array}{c}\text { Anorexie mentale } \\
+ \text { Boulimie [bulimia nervosa] }\end{array}$ \\
\hline 7.11 & Anorexie mentale atypique & F50.1 & Anorexie mentale atypique \\
\hline 7.12 & Boulimie & $\mathrm{F} 50.2$ & Boulimie[bulimia nervosa] \\
\hline 7.13 & Boulimie atypique & $\mathrm{F} 50.3$ & Boulimie atypique \\
\hline 7.14 & $\begin{array}{l}\text { Tr. des conduites alimentaires du nourrisson et de } \\
\text { l'enfant }\end{array}$ & F98.2 & $\begin{array}{l}\text { Trouble de l'alimentation non organique du nourrisson } \\
\text { et de l'enfant }\end{array}$ \\
\hline 7.15 & Troubles alimentaires du nouveau-né & F98.2 & $\begin{array}{l}\text { Trouble de l'alimentation non organique du nourrisson } \\
\text { et de l'enfant }\end{array}$ \\
\hline 7.18 & Autres tr. des conduites alimentaires & F50.8 & Autres troubles de l'alimentation \\
\hline 7.19 & Tr. des conduites alimentaires NS & F50.9 & Trouble de l'alimentation, sans précision \\
\hline 7.2 & CONDUITES SUICIDAIRES & & \\
\hline$\underline{7.20}$ & Tentatives de suicide suite immédiate & Z91.50 & $\begin{array}{l}\text { Antécédents personnels récents de lésions auto- } \\
\text { infligées }\end{array}$ \\
\hline$\underline{7.21}$ & Antécédents de TdS & Z91.58 & $\begin{array}{c}\text { Antécédents personnels de lésions auto-infligées, } \\
\text { autres et non précisés }\end{array}$ \\
\hline$\underline{7.22}$ & Idées suicidaires & R45.8 & Autres symptômes et signes relatifs à l'humeur \\
\hline 7.3 & TROUBLES LIÉS À L'L & AGE DE & ROGUES OU D'ALCOOL* \\
\hline $7.3 \times 9$ & Alcool* & F10.- & $\begin{array}{l}\text { Troubles mentaux et du comportement liés à l'utilisation } \\
\qquad \text { d'alcool* }^{*}\end{array}$ \\
\hline $7.3 \times 0$ & - Morphiniques* & F11.- & $\begin{array}{l}\text { Troubles mentaux et du comportement liés à l'utilisation } \\
\text { d'opiacés* }\end{array}$ \\
\hline $7.3 \times 1$ & - Cannabis* & F12.- & $\begin{array}{l}\text { Troubles mentaux et du comportement liés à l'utilisation } \\
\text { de dérivés du cannabis* }\end{array}$ \\
\hline $7.3 \times 2$ & - Hypnotiques et tranquillisants* & F13.- & $\begin{array}{l}\text { Troubles mentaux et du comportement liés à l'utilisation } \\
\text { de sédatifs ou d'hypnotiques* }\end{array}$ \\
\hline $7.3 \times 3$ & - Cocaïne* & F14.- & $\begin{array}{l}\text { Troubles mentaux et du comportement liés à l'utilisation } \\
\qquad \text { de cocaïne* }\end{array}$ \\
\hline $7.3 \times 4$ & - Autres stimulants* & F15.- & $\begin{array}{l}\text { Troubles mentaux et du comportement liés à l'utilisation } \\
\text { d'autres stimulants, y compris la caféine* }\end{array}$ \\
\hline $7.3 \times 5$ & - Hallucinogènes* & F16.- & $\begin{array}{l}\text { Troubles mentaux et du comportement liés à l'utilisation } \\
\text { d'hallucinogènes* }\end{array}$ \\
\hline $7.3 \times 6$ & - Tabac* & F17.- & $\begin{array}{l}\text { Troubles mentaux et du comportement liés à l'utilisation } \\
\qquad \text { de tabac* }\end{array}$ \\
\hline
\end{tabular}




\begin{tabular}{|c|c|c|c|}
\hline $7.3 \times 7$ & - Solvants volatils* & F18.- & $\begin{array}{l}\text { Troubles mentaux et du comportement liés à l'utilisation } \\
\text { de solvants volatils* }\end{array}$ \\
\hline $7.3 \times 8$ & - Autres substances psychoactives* & F19.- & $\begin{array}{c}\text { Troubles mentaux et du comportement liés à l'utilisation } \\
\text { de drogues multiples et troubles liés à l'utilisation } \\
\text { d'autres substances psychoactives* }\end{array}$ \\
\hline & * Préciser le type de trouble mental : & & * Préciser le type de trouble mental : \\
\hline $7.30 \mathrm{X}$ & Intoxication aigüe & 0 & Intoxication aiguë \\
\hline $7.31 \mathrm{X}$ & Utilisation nocive pour la santé & .1 & Utilisation nocive pour la santé \\
\hline $7.32 X$ & Syndrome de dépendance & .2 & Syndrome de dépendance \\
\hline $7.33 \mathrm{X}$ & Syndrome de sevrage & .3 & Syndrome de sevrage \\
\hline $7.34 \mathrm{X}$ & Syndrome de sevrage avec delirium & .4 & Syndrome de sevrage avec délirium \\
\hline $7.35 \mathrm{X}$ & Trouble psychotique & .5 & Trouble psychotique \\
\hline $7.36 \mathrm{X}$ & Syndrome amnésique & .6 & Syndrome amnésique \\
\hline $7.37 \mathrm{X}$ & Troubles. résiduel ou psychotique tardif & .7 & Trouble résiduel ou psychotique tardif \\
\hline $7.38 \mathrm{X}$ & Autres tr. mentaux et du comportement & .8 & Autres troubles mentaux et du comportement \\
\hline 7.39X & $\begin{array}{l}\text { Troubles. mental ou du comportement sans } \\
\text { précision }\end{array}$ & .9 & Trouble mental ou du comportement sans précision \\
\hline 7.4 & TR. DE L'ANGOISSE DE SÉPARATION & F93.0 & Angoisse de séparation de l'enfance \\
\hline 7.5 & \multicolumn{3}{|c|}{ TR. DE L'IDENTITÉ ET DES CONDUITES SEXUELLES } \\
\hline 7.50 & Troubles de l'identité sexuelle & F64.2 & Trouble de l'identité sexuelle de l'enfance \\
\hline 7.51 & Troubles de la préférence sexuelle & F65.9 & Trouble de la préférence sexuelle, sans précision \\
\hline 7.52 & $\begin{array}{l}\text { Manifestations en rapport avec des préoccupations } \\
\text { excessives concernant le développement sexuel et } \\
\text { son orientation }\end{array}$ & F66.0 & Trouble de la maturation sexuelle \\
\hline 7.58 & Autres tr. des conduites sexuelles & F66.8 & Autres troubles du développement psychosexuel \\
\hline 7.59 & Troubles. des conduites sexuelles NS & F66.9 & $\begin{array}{l}\text { Trouble du développement psychosexuel, sans } \\
\text { précision }\end{array}$ \\
\hline 7.6 & PHOBIES SCOLAIRES & F94.8 & $\begin{array}{l}\text { Autres troubles du fonctionnement social } \\
\text { de l'enfance }\end{array}$ \\
\hline 7.7 & AUTRES TR. CARACTERISÉS DES CONDU & & \\
\hline 7.70 & Pyromanie & F63.1 & $\begin{array}{l}\text { Tendance pathologique à allumer des incendies } \\
\text { [pyromanie] }\end{array}$ \\
\hline
\end{tabular}




\begin{tabular}{|c|c|c|c|}
\hline 7.71 & Kleptomanie & F63.2 & $\begin{array}{c}\text { Tendance pathologique à commettre des vols } \\
\text { [kleptomanie] }\end{array}$ \\
\hline 7.72 & Trichotillomanie & F63.3 & Trichotillomanie \\
\hline 7.73 & Fugues & F91.8 & Autres troubles des conduites \\
\hline 7.74 & Violence contre les personnes & R45.6 & Violence physique \\
\hline 7.75 & Conduites à risques & F91.8 & Autres troubles des conduites \\
\hline 7.76 & Errance & F91.8 & Autres troubles des conduites \\
\hline 7.78 & Autres tr. caractérisé des conduites & F91.8 & Autres troubles des conduites \\
\hline 7.8 & AUTRES TR. DES CONDUITES ET DES & F91.8 & Autres troubles des conduites \\
\hline 7.9 & TR. DES CONDUITES ET DES COMPORTEMENTS & $\mathrm{F91.9}$ & Trouble des conduites, sans précision \\
\hline
\end{tabular}




\begin{tabular}{|c|c|c|c|}
\hline 8 & TROUBLES À EXPRESSION & & \\
\hline 8.0 & AFFECTIONS PSYCHOSOMATIQUES & F45.0 & Somatisation \\
\hline 8.1 & TR. PSYCHOFONCTIONNELS & F45.- & Troubles somatoformes \\
\hline 8.2 & TR. HYPOCONDRIAQUES & F45.2 & Troubles hypocondriaque \\
\hline 8.3 & ÉNURESIE & F98.0 & Enurésie non organique \\
\hline 8.4 & ENCOPRESIE & F98.1 & Encoprésie non organique \\
\hline 8.5 & TR. DU SOMMEIL & F51.9 & Troubles du sommeil non organique, sans \\
& & & précision \\
\hline 8.6 & RETARD DE CROISSANCE PSYCHOGENE & F94.8 & Autres troubles du fonctionnement social \\
& & + R62.9 & de l'enfance \\
\hline 8.8 & AUTRES TR. À EXPRESSION SOMATIQUE & F45.8 & $\begin{array}{c}\text { Autres troubles somatoformes } \\
\text { + Retard du développement }\end{array}$ \\
\hline
\end{tabular}

\begin{tabular}{|c|c|c|c|}
\hline \multicolumn{3}{|c|}{ MANIFESTATIONS ET SYMPTOMES } & \\
\hline 9.0 & SYVERS & & \\
\hline 9.00 & Attaques de panique & & \\
\hline 9.01 & Anxiété généralisée & F41.0 & Troubles panique [anxiété épisodique paroxystique] \\
\hline 9.08 & Avec autres manifestations anxieuses & F41.8 & Anxiété généralisée \\
\hline 9.1 & SYMPTOMES CONVERSIFS & & Autres troubles anxieux précisés \\
\hline 9.10 & Symptômes moteurs de conversion & F44.4 & Troubles moteurs dissociatifs \\
\hline 9.01 & Symptômes sensoriels de conversion & F44.6 & Anesthésie dissociative et atteintes sensorielles \\
\hline 9.02 & Multiples symptômes de conversion & F44.7 & Trouble dissociatif [de conversion] mixte \\
\hline 9.08 & Autres symptômes de conversion & F44.8 & Autres troubles dissociatifs [de conversion] \\
\hline
\end{tabular}




\begin{tabular}{|c|c|c|c|}
\hline 9.2 & SYMPTOMES PHOBIQUES & F91.8 & Autres troubles des conduites \\
\hline 9.20 & Avec symptômes agoraphobiques & F40.0 & Agoraphobie \\
\hline 9.200 & Agoraphobie sans trouble panique & F40.00 & Agoraphobie sans trouble panique (actuel) \\
\hline 9.201 & Agoraphobie avec trouble panique & F40.01 & Agoraphobie avec trouble panique \\
\hline 9.21 & Phobies sociales & F40.1 & Phobies sociales \\
\hline 9.22 & Phobies scolaires & F94.8 & Autres troubles du fonctionnement social de l'enfance \\
\hline 9.23 & Phobies spécifiques (isolées) & F40.2 & Phobies spécifiques (isolées) \\
\hline 9.28 & Autres symptômes phobiques & F40.8 & Autres troubles anxieux phobiques \\
\hline 9.3 & $\begin{array}{c}\text { MANIFESTATIONS OBSESSIONNELLES ET } \\
\text { COMPULSIVES (TOC) }\end{array}$ & & \\
\hline 9.30 & TOC idées obsédantes au premier plan & F42.0 & $\begin{array}{l}\text { TOC avec idées ou ruminations obsédantes au premier } \\
\text { plan }\end{array}$ \\
\hline 9.31 & TOC rituels compulsifs au premier plan & F42.1 & $\begin{array}{c}\text { TOC avec comportements compulsifs [rituels } \\
\text { obsessionnels] au premier plan }\end{array}$ \\
\hline 9.32 & TOC forme mixte & F42.2 & $\begin{array}{c}\text { TOC : forme mixte, avec idées obsédantes et } \\
\text { comportements compulsifs }\end{array}$ \\
\hline 9.38 & Autres TOC & F42.8 & Autres TOC \\
\hline
\end{tabular}


Tableau 2

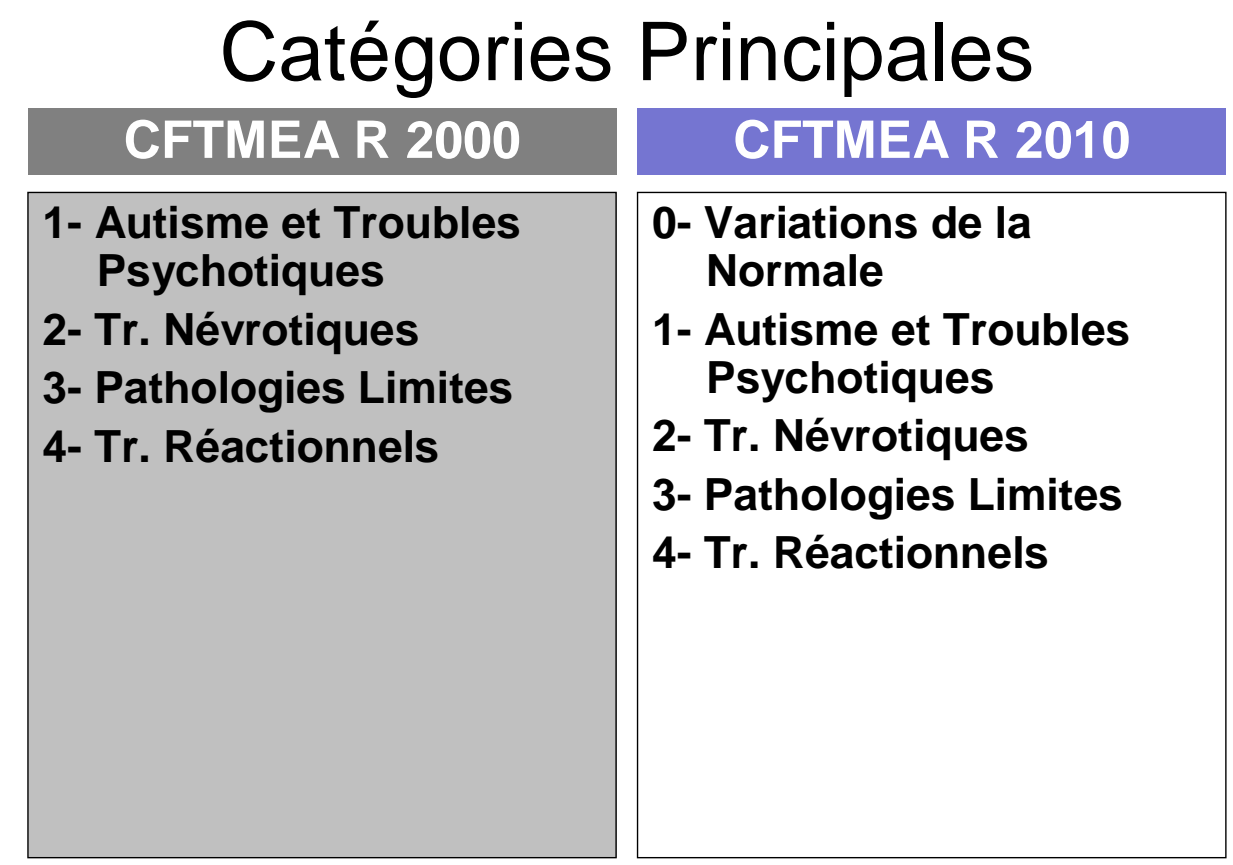

Tableau 1: les catégories principales de la CFTMEA

Tableau 3 


\section{Catégories Complémentaires}

\section{CFTMEA R 2000}

5- Déficiences Mentales

6- Tr. du Développement et des Fonctions Instrumentales

7- Tr. des Conduites \& du Comportement

8- Tr. à Expression Somatique

9- Variations de la Normale

\section{CFTMEA R 2010}

5- Déficiences Mentales

6- Tr. du Développement et des Fonctions Instrumentales

7- Tr. des Conduites \& du Comportement

8- Tr. à Expression Somatique

9- Manifestations et Symptômes Divers

Tableau 1: les catégories complémentaires de la CFTMEA 